BULLETIN OF THE

AMERICAN MATHEMATICAL SOCIETY

Volume 79, Number 1, January 1973

\title{
STABILITY IMPLIES NORMAL AND DISC BUNDLES ${ }^{1}$
}

\author{
BY RONALD J. STERN ${ }^{2}$
}

Communicated by E. Spanier, June 16, 1972

Introduction. In this note we announce results concerning normal bundles, disc bundles, and Stiefel-Whitney classes in the topological category. Many of these results also hold in the piecewise linear (PL) category, but the dimensions should be restricted accordingly.

I would like to thank Professors R. F. Brown and R. Edwards for many helpful and encouraging discussions.

Normal bundles and disc bundles. Let $\mathrm{TOP}_{n}$ be the semisimplicial (s.s.) group of topological origin-preserving homeomorphisms of $R^{n}$. Let $\mathrm{TOP}_{n, k}$ be the s.s. group of topological homeomorphisms of $R^{n}=$ $R^{n-k} \times R^{k}$ which are pointwise fixed on $R^{k}$.

In [9] Kirby and Siebenmann announced a strong stability theorem for TOP $/ O$, i.e., if $n \geqq 5$, the stability map

$$
s_{i}: \pi_{i}\left(\mathrm{TOP}_{n}, O_{n}\right) \rightarrow \pi_{i}\left(\mathrm{TOP}_{n+1}, O_{n+1}\right)
$$

is an isomorphism for $i \leqq n+1$ and an epimorphism for $i=n+2$, where $O_{n}$ is the s.s. $n$-dimensional orthogonal group. Using this result we deduce that

THEOREM 1. $\pi_{i}\left(\operatorname{TOP}_{n}, \operatorname{TOP}_{n}(I)\right)=0$ for $i \leqq n+1, n \geqq 6$, where $\operatorname{TOP}_{n}(I)$ is the s.s. group of topological origin-preserving homeomorphisms of the unit disc in $R^{n}$.

An immediate corollary is

Corollary 2. Let $X$ have the homotopy type of a k-dimensional $C W$ complex. Any $R^{n}$-bundle over $X$ contains a disc bundle if $n \geqq k-2, n \geqq 6$. It is uniquely determined (up to isomorphism) if $n \geqq k-1, n \geqq 6$.

In particular, every $n$-manifold, $n \geqq 6$, has a tangent disc bundle.

Using the above stability result and results of Rourke and Sanderson ([12], [13]), we show that

THEOREM 3. $\pi_{i}\left(\mathrm{TOP}_{n, k}, \mathrm{TOP}_{n-k}\right)=0$ if $i \leqq n-k+j, n-k \geqq 5+j$, $j=0,1,2$.

AMS (MOS) subject classifications (1970). Primary 57A40, 55F60, 57E05, 55F10; Secondary 55G40, 55F 40 .

Key words and phrases. Normal bundle, disc bundle, Stiefel-Whitney classes, $R^{n}$-bundle

${ }^{1}$ These results will appear in the author's doctoral thesis at UCLA.

${ }^{2}$ Research supported in part through an NSF Traineeship at UCLA. 
Then, by the results of K. Millett [10], Browder [2], and Theorem 3, one can show the existence of disc bundles in Corollary 2 for $R^{n}$-bundles with nonzero cross section when $n=k-3, n \geqq 11$.

Using Theorem 3 and the immersion theorem of Lees or Gauld [7] we immediately obtain

COROLlARY 4. Let $M^{n}$ be a locally flat submanifold of $N^{n+q}$. If $q \geqq 5+i$, then $M$ has a normal open or microbundle in $N$ if $q \geqq n-i-1, i=0,1,2$. If $q \geqq n-i$ it is unique up to isotopy.

REMARK. We obtain three more dimensions for disc bundles and open normal bundles than [13].

Stiefel-Whitney classes. Let $\xi=\left(E, E_{0}, p, B\right)$ be an $R^{n}$-bundle with zero-section, where $E_{0}=E$-zero section, $p: E \rightarrow B$, and $B$ is compact having the homotopy type of an $m$-dimensional $\mathrm{CW}$-complex. Let $w_{i}(\xi)$ denote the $i$ th Stiefel-Whitney class of $\xi$. The top class of $\xi$, denoted $W_{n}(\xi)$, is given by $W_{n}(\xi)=\Phi^{-1}\left(U_{\xi} \cup U_{\xi}\right)$, where $U_{\xi} \in H^{n}\left(E, E_{0} ; p^{*} \Gamma\right)$ is the Thom class, $\Gamma$ the local system of $B$ such that

$$
\Gamma(b)=H_{n}\left(p^{-1}(b), p_{0}^{-1}(b) ; Z\right),
$$

and $\Phi$ the Thom isomorphism.

Let $\xi\left(V_{n, k}^{\prime}\right)$ be the bundle associated to $\xi$ with fiber $\operatorname{TOP}_{n} / \operatorname{TOP}_{n-k}$. Let $c_{n-k+1}(\xi)$ denote the primary obstruction to finding a cross section of $\xi\left(V_{n, k}^{\prime}\right)$. Using Theorem 1, results of Akiba [1], and variations of classical techniques in the differentiable category we prove

THEOREM 5. If $n \neq 4,5, n \geqq m-2$, then $c_{n}(\xi)=0$ iff $W_{n}(\xi)=0$. If $n-k \geqq 5, c_{n-k+1}(\xi)$ reduced $\bmod 2$ equals $w_{n-k+1}(\xi)$. Also $c_{n}(\xi)=\lambda W_{n}(\xi)$ where $\lambda=0$ iff $W_{n}(\xi)=0$.

Let $\xi\left(V_{n, k}\right)$ be the bundle associated to $\xi$ with fiber $\mathrm{TOP}_{n} / \mathrm{TOP}_{n, k}$. Let $d_{n-k+1}(\xi)$ denote the primary obstruction to finding a cross section to $\xi\left(V_{n, k}\right)$. Using a theorem of K. C. Millett [10] and Theorem 3, we show that

THEOREM 6. If $n \neq 4, m+5 \leqq 2(n-k)$, then $d_{n}(\xi)=0$ iff $W_{n}(\xi)=0$. If $n-k \geqq 5$, then $d_{n-k+1}(\xi)$ reduced $\bmod 2$ equals $w_{n-k+1}(\xi)$. Also $d_{n}(\xi)=\lambda W_{n}(\xi)$ where $\lambda=0$ iff $W_{n}(\xi)=0$.

These theorems provide a geometrical interpretation of the StiefelWhitney classes in the topological category similar to that in the differentiable category (see $[\mathbf{1 1}],[\mathbf{1 4}]$ ).

REMARK. We can also prove results analogous to Theorems 4 and 5 in the PL category with no restrictions on $n-k$, by using results of Hirsch [8]. However, in Theorem 5 we must have $n \geqq m$. 
Let $M^{n}$ be a compact $n$-manifold with or without boundary. An arcfield on $M$ is a map $p: M \rightarrow M^{I}$ such that, for all $b \in M, p(b)(0)=b$ and $p(b)$ is a homeomorphism. Using results of R. F. Brown and E. Fadell [3], Hirsch [8], and Theorem 6 we make the following observation.

COROLlary 7. If $M^{n}$ is a PL-manifold, or if $n \neq 4, M$ has an arc-field iff the Euler characteristic of $M$ is zero.

This was proven for all triangulated manifolds by Fadell [5].

REMARK. In view of Theorems 5 and 6 the appropriate topological version of a tangent $k$-field should be a map $p: M \rightarrow M^{R^{k}}$ where, for all $b \in M, p(b)(0)=b$ and $p(b)$ is a locally flat homeomorphism. This answers a question posed by E. Fadell [6].

Details of proofs will appear elsewhere.

\section{REFERENCES}

1. T. Akiba, The homotopy type of some PL complexes, Bull. Amer. Math. Soc. 77 (1971), 1060-1062.

2. W. Browder, Open and closed disc bundles, Ann. of Math. (2) 83 (1966), 218-230. MR 32 \#6485.

3. R. F. Brown and E. Fadell, Nonsingular path fields on compact topological manifolds, Proc. Amer. Math. Soc. 16 (1965), 1342-1349. MR 32 \# 1709.

4. E. Fadell, Generalized normal bundles for locally-flat imbeddings, Trans. Amer. Math. Soc. 144 (1965), 488-513. MR 31 \# 4037.

5. - A remark on simple path fields in polyhedra of characteristic zero, Rocky Mountain J. Math. (to appear).

6. - Some questions in the theory of normal fiber spaces for topological manifolds, Topology Seminar, Wisconsin, 1965, edited by R. Bing and R. Bean, Ann. of Math. Studies, No. 60, Princeton Univ. Press, Princeton, N.J.

7. D. Gauld, Mersions of topological manifolds, Trans. Amer. Math. Soc. 149 (1970), 539-560. MR 42 \# 1124.

8. M. W. Hirsch, On tubular neighborhoods of piecewise linear and topological manifolds, Conference on the Topology of Manifolds (Michigan State Univ., E. Lansing, Mich., 1967),

Prindle, Weber and Schmidt, Boston, Mass., 1968, pp. 63-80. MR 38 \# 5226.

9. R. Kirby and L. Siebenmann, Some theorems on topological manifolds, Lecture Notes in Math., vol. 197, Springer-Verlag, Berlin and New York, 1971.

10. K. Millett, Nonsingular sections to Euclidean bundles, Trans. Amer. Math. Soc. 144 (1969), 127-143. MR 40 \# 6571.

11. J. Milnor, Lectures on characteristic classes, Princeton University, Princeton, N.J., 1957 (mimeographed notes).

12. - Block bundles. III. Homotopy theory, Ann. of Math. (2) 87 (1968), 431-483. MR 38 \# 729 .

13. C. P. Rourke and B. J. Sanderson, On topological neighborhoods, Compositio Math. 22 (1970), 387-424.

14. N. E. Steenrod, The topology of fibre bundles, Princeton Math. Series, vol. 14, Princeton Univ. Press, Princeton, N.J., 1951. MR 12, 522.

Department of Mathematics, University of California, los Angeles, California 90024 